\title{
Developing a trustworthy quality improvement framework
}

\author{
Author: Jonathan Mamo
}

\author{
Aims \\ To develop a dynamic and facilitated quality improvement (QI) \\ project framework within Solent NHS Trust for doctors-in-training \\ to improve junior doctor engagement with quality and service \\ improvement projects.
}

\section{Methods}

The NHS is currently showing a gradual shift towards further quality and service improvement projects and we highlighted the trust's need for improved support for doctors-in-training. The requirement for a quality improvement project (QIP) to be mandated as a part of all trainees' portfolio indicated a need for the trust to provide facilitation and support in the process.

The chief registrar-led project involved evaluation of prior facilities and support for doctors requesting to lead or involve themselves in a QIP. This included investigation of other trusts' experience in implementing similar frameworks. The trust commenced offering training and educational opportunities to newly inducted doctors with optional drop-in sessions as support. Project registration was also included to provide ease of communication with a plan for future project database creation.

\section{Results}

The number of QIPs at 6 months increased from none to five doctor-led projects. This equated to over a quarter of the trust's doctors-in-training being involved in a QIP. Two of the QIPs were presented at a trust showcase event. One of the QIPs showed a very conservative cost saving of $£ 40,000$ when taking staff time only into consideration. This number of projects continues to grow as each new cohort of doctors-in-training takes on new QIPs with QI team assistance.

\section{Conclusion}

Continued QIP support and education is to be introduced to all newly inducted doctors. Eventual project database would be available on the junior doctor intranet page. The results of future QIPs could be used to calculate cost savings for the trust. The initial QIP framework was targeted towards doctors-in-training but after

Author: Royal College of Physicians chief registrar, Solent NHS Trust, UK further consultation with the consultant team it was to be rolled out to any interested doctors working within Solent NHS Trust.

\section{Conflict of interest statement}

No conflict of interest. 